\title{
Pain, pleasure and placebo: the cannabinoids in reward processing and the perception of pain
}

\author{
Maximillian H. Brodermann* \\ University of Oxford, Oxford, United Kingdom
}

\begin{abstract}
Chronic pain poses a complex, widespread problem that remains inadequately resolved by pain medication. Similarly, depression and addiction exist along a spectrum of reward related mood disorders that pose an ever-increasing burden on healthcare services. There is a need for a greater range of drugs to tackle these diverse health conditions and cannabinoid biology offers a unifying line of research that could invigorate the approach to treatment. Cannabinoids are known contributors to the physiological basis and emotional perception of pain, pleasure, and the placebo effect via activation of cannabinoid type 1 receptors widely expressed throughout the central nervous system. Endogenous cannabinoids - arachidonic acid derivatives synthesised on demand - are the primary contributors, but the system is also susceptible to synthetic cannabinoids and plant-derived phytocannabinoids. Targeting the endocannabinoid system pharmacologically has yielded few licenced drugs and with limited applications. The failure to provide a greater range of drugs stems partly from the limited understanding of endogenous cannabinoid biology. This review will critically evaluate current evidence of how cannabinoids influence pain, hedonic reward processing, and the placebo effect. Crucially, the endocannabinoid system must not be considered in isolation, but in the context of opioid and dopaminergic neural circuitry. As such, the most exciting pharmacological opportunities could lie as adjuncts to pre-existing drugs.
\end{abstract}

\section{Introduction}

Pain is the negative sensory and affective response to noxious or potentially harmful stimuli relayed to the brain. By contrast, pleasure is what drives reward seeking behaviour. Although pain and pleasure are contrasting emotional states, they share neurobiological pathways and strongly influence each other: pleasure increases our ability to tolerate pain, and pain lowers our capacity to experience pleasure [1]. Moreover, by hijacking their common neural networks, the placebo effect powerfully modulates how we perceive both pleasure and pain [2].

The endogenous opioid system is thought to play a central role in the pain, pleasure, placebo overlap [1], but evidence presented in this review suggests that cannabinoids are equally important mediators and offer a host of attractive therapeutic opportunities as wide ranging as anxiety and addiction. The existence of cannabinoids has been known for millennia [3], with particular interest surrounding $\Delta$-9-tetrahydrocannabinol (THC), the active ingredient of cannabis. However, only recently has the widespread significance of cannabinoid biology in neurological function and its potential in contemporary medicine been explored. At a time when the legalisation of cannabis and its derivatives is increasingly debated and accepted, it is more important than ever to understand the benefits and potential harms presented by both endogenous and exogenous cannabinoids. This brief review will focus on how cannabinoid biology associates with fundamental pain and reward neural circuitry and consider the potential impact of further discoveries in the field.

\section{Overview of cannabinoid biology}

The endocannabinoid system (ECS) outlined in Figure 1 is a highly conserved network known to modulate a wide range of physiological processes including nociception, metabolism, and immune function
[4-7]. Interest in the cannabinoids originally stemmed from the psychoactive effects of THC. Although THC was first isolated in 1964 [3], it took a further 24 years to discover its endogenous receptor [8]. There are now two known G-protein coupled cannabinoid receptors: cannabinoid receptor type $1\left(\mathrm{CB}_{1}\right)$ predominantly expressed in the brain and on peripheral neurons, and cannabinoid receptor type $2\left(\mathrm{CB}_{2}\right)$, expressed throughout the immune system with a functional significance that is still under active investigation. The endogenous ligands anandamide and 2-arachidonoylglycerol (2AG) are synthesised de-novo from lipid precursors and have short half-lives due to rapid degradation by fatty acid amide hydrolase (FAAH) and monoacylglycerol lipase (MAGL) respectively [9]. Both endocannabinoids are agonists at $\mathrm{CB}_{1}$ and $\mathrm{CB}_{2}$, signalling via mitogenactivated protein kinases (MAPK) and inhibition of adenylyl cyclase to lower intracellular cAMP concentrations, as summarised in Figure 1. The generation of $\mathrm{CB}_{1}$ and $\mathrm{CB}_{2}$ inverse agonists, FAAH and MAGL inhibitors, and synthetic $\mathrm{CB}_{1}$ and $\mathrm{CB}_{2}$ agonists has provided tools to study the importance of the ECS in the pain, pleasure, and placebo axis.

\section{No pain, no gain}

Ultimately, pain, pleasure, and placebo are all multifaceted and subjective experiences, heavily influenced by the patient's affective state. It follows that pain could be alleviated by manipulating the belief that pain is indeed adverse. Benedetti et al. tested this by inducing

Correspondence to: Max Brodermann, Hertford College, Catte Street, University of Oxford, OX1 3BW, United Kingdom, Tel: +44 (0) 7787766279; E-mail:max. brodermann@hertford.ox.ac.uk

Key words: Pain, Placebo, Pleasure, Cannabinoid, Opioid, Dopamine

Received: September 12, 2016; Accepted: September 23, 2016; Published: September 26, 2016 


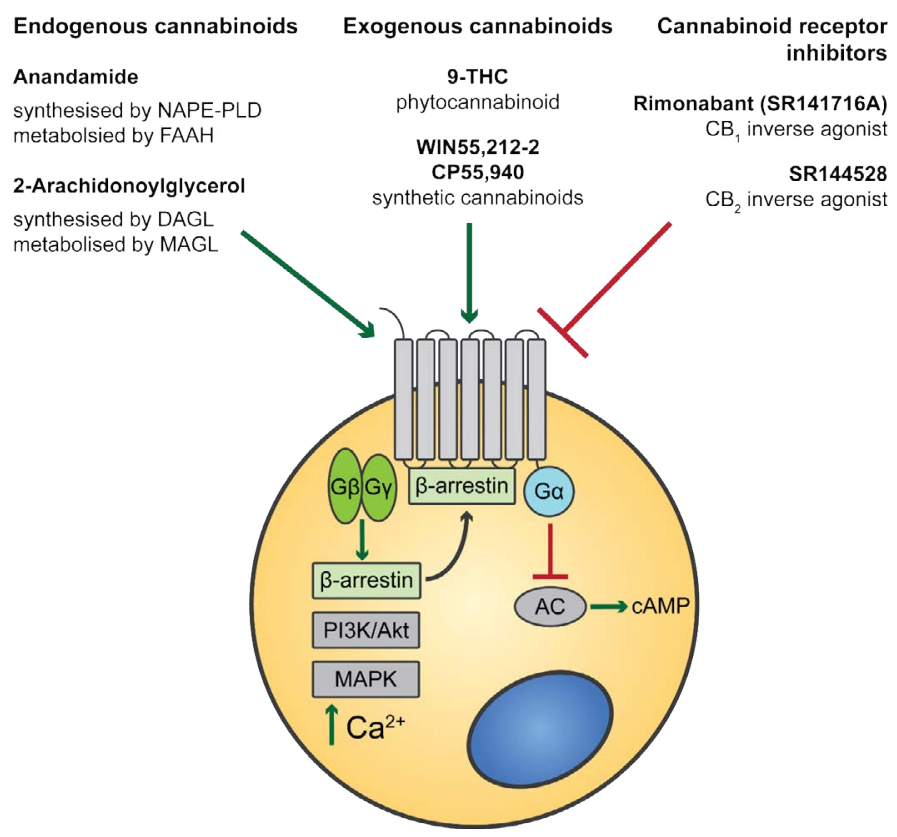

Figure 1. An overview of cannabinoid biology and signalling pathways. Endogenous and exogenous cannabinoids activate $\mathrm{CB}_{1}$ and $\mathrm{CB}_{2}$ and signal through MAPK and inhibition of adenylyl cyclase $(\mathrm{AC})$. NAPE-PLD $=\mathrm{N}$-acyl phosphatidylethanolamine phospholipase $\mathrm{D}, \mathrm{FAAH}=$ Fatty acid amide hydrolase, $\mathrm{DAGL}=$ Diacylglycerol lipase, $\mathrm{MAGL}=$ Monoacylglycerol lipase.

ischaemic arm pain in two groups of volunteers by vertically extending volunteers' arms to drain the venous blood, applying a tourniquet, and asking subjects to squeeze a hand spring exerciser in two second intervals for as long as possible [10]. The negative group was told that the tourniquet test would cause pain with no beneficial side effects; the positive group was told that the pain would benefit their muscle fibre capabilities. Collectively, the positive group showed significantly higher pain tolerance to the tourniquet test. This response is characteristic of placebo analgesia, whereby positive beliefs and expectations are beneficial to the outcome of the patient. The objective discomfort of ischaemic arm pain was masked by the reward of improved muscle performance - akin to forms of exercise. When the positive group was administered rimonabant $\left(\mathrm{CB}_{1}\right.$ inverse agonist) or naltrexone (opioid antagonist), the rise in pain tolerance was attenuated. Administered together, the rise in pain tolerance was eliminated altogether. These observations suggest that cannabinoids and opioids act synergistically to mediate placebo analgesia.

The results from this investigation substantiate the potential for placebos in a clinical setting to help dissociate pain from its negative connotations. Benedetti et al. suggest that cannabinoid signalling contributes to placebo analgesia, buthumans that carry theFAAHC $385 \mathrm{~A}$ missense mutation, and thus have increased basal levels of anandamide, show paradoxically reduced placebo analgesia responses [11]. A simple explanation is that elevated concentrations of endocannabinoids cause $\mathrm{CB}_{1}$ receptor desensitisation. Alternatively, preconditioning to a pain challenge could alter the balance of neurobiological mediators involved in placebo analgesia. Wiech et al. propose that conditioning prior to a pain stimulus involves 'perceptual decision making' [12]: modulating the sensory input according to comprehension of pain stimuli based on previous experience, possibly by activating the anterior cingulate cortex and the dorsolateral prefrontal cortex, both implicated in decision making [12]. Adopting Benedetti et al. study design to include and account for individuals carrying the FAAH C385A mutation may help delineate the extent of opioid, cannabinoid and associated signalling events in placebo analgesia.

\section{Endocannabinoids in reward processing}

From an evolutionary perspective, animals are motivated by rewards that promote survival. We subsist on feeding, social interaction, and sexual intercourse and therefore attribute hedonic reward value to such behaviour. Being in a positive affective state can also reduce the perception of pain [13] - a phenomenon called pleasure related analgesia (PRA). Opioid and cannabinoid signalling are involved in responding to pleasurable stimuli [14-16], but their relative contribution to PRA was unresolved until recently.

Kut et al. [17] hypothesised that PRA would be brought about by increased opioidergic signalling. Pain tolerance was measured before and after subjects viewed pleasant pictures from the International Affective Picture System (IAPS). Pain tolerance increased after viewing the pictures, an effect that persisted following opioid blockade with naloxone. This suggests that an opioid-insensitive circuit may mediate PRA.

A follow up study by Friemel et al. [18] built upon previous studies reporting the role of the ECS in motivational behaviour and operant conditioning [19], hypothesising that endocannabinoid signalling may be involved in evaluating the hedonic component of a reward. To test this, the group employed a paradigm assessing the attenuation of the acoustic startle reflex (ASR) when rodents were in a hedonic state, the so-called pleasure-attenuated startle (PAS). This hedonic state was induced by an odour learned to be associated with a sweetened condensed milk (SCM) reward. Using the odour as a neutral affective stimulus associated with a physical reward is likely to be a more powerful pleasure inducer than the purely psychological reward obtained from positive IAPS pictures. In trained animals, the odour (without the presence of the SCM reward) was sufficient to reduce the startle reflex. This is an elegant experimental paradigm as it measures the change of a physiological reflex in a pleasurable state when the physical reward itself is not present and only the attributed value of the reward can drive the reduction in ASR, not the conditioned behaviour of obtaining the reward when it is there. To validate the model, a second group of rodents were sham trained with SCM rewards without the odour present and showed no significant increase in PAS in the presence of the odour during the investigation.

To assess whether the ECS was mediating PAS in rats, the group administered the cannabinoid agonist WIN55,212-2 and rimonabant in independent experiments. WIN55,212-2 caused a significant increase in PAS, whereas rimonabant caused a significant decrease in PAS compared to vehicle, consistent with the hypothesis that endocannabinoid signalling mediates reward processing. With the protocol established in rats, and seeking to obtain further information about the nature of endocannabinoid signalling, the group generated $\mathrm{CB}_{1}$ knockout $(\mathrm{KO})$ mice and tested PAS with the same startle protocol used in rats. Compared to wild-type (WT) mice, $\mathrm{CB}_{1} \mathrm{KO}$ mice had a significantly lower, indeed absent, PAS. Collectively, this genetic and pharmacological data comprehensively implicates $\mathrm{CB}_{1}$ in reward processing, not simply driving operant behaviour but actively signalling the pleasure of a reward.

This study informs how compulsive behaviour and addiction could be treated. Compared to our distant ancestors, we no longer have the basic survival needs of food, water, and social interaction. The word 'reward' has progressed to encompass desires that do not necessitate 
survival but are nonetheless considered pleasurable. Less primal, higher order desires such as travel, sport, music, refined carbohydrates, and addictive drugs all activate neural reward pathways [20]. Ascribed reward value is derived from our reinforced expectation of a given outcome [20], and can be manipulated by placebos operating through the same neurochemical pathways as the active drug itself [21]. Studies of the psychostimulant methylphenidate [22] indicate that the expectation of receiving the drug (versus a placebo) can increase the effect of the drug itself by $50 \%$, as measured by increases in brain glucose metabolism via positron emission topography (PET). Gu et al. [23] reported a similar finding with the expectation of nicotine, which suggests that placebo therapy targeting the 'concomitant set of sensory and social stimuli' [21] that underpin the perceived benefit of a reward could be instrumental in treating aspects of addiction.

The motivating factors that lead to addiction differ between individuals, but whether an addictive substance is pursued to enhance pleasure or reduce pain may not be of significance as either is perceived as rewarding. The neural circuitry mediating the hedonic value of rewards includes foci of 'hedonic hotspots' such as the nucleus accumbens and ventral pallidum [20]. The latter is of particular significance in being the only known area where focal lesions can reverse the feeling of pleasure to a sense of revulsion for the same given stimulus [24]. This unique facet coupled with the ventral pallidum's relatively high density of $\mathrm{CB}_{1}$ renders it an attractive target for modulating reward pathways in response to drivers of addiction [25].

\section{Cannabinoid co-operation in reward processing}

Dopaminergic neurotransmission is fundamental to the mesolimbic and mesocortical circuits that predominate in reward cognition and reinforcement learning to control goal-directed behaviour [19]. Just as there is an inextricable link between cannabinoids and opioids in the mediation of pain, there is a strong overlap between cannabinoids and dopamine (DA) in the mediation of pleasure. An example of their interdependence is the observation that DA neurons express diacylglycerol-lipase- $\alpha$ (DAGL- $\alpha$ ) and can generate 2-AG that acts presynaptically to inhibit GABAergic and glutamatergic input to DA neurons [19]. This is defined as depolarisation-induced suppression of inhibition (DSI) or depolarisation-induced suppression of excitation (DSE). Disrupting the delicate interplay between cannabinoids and dopaminergic signalling, specifically through exogenous cannabinoids, can greatly disrupt learning and goal directed behaviour. For example, cannabis users show learning and memory deficits through frontocortical hypoactivity and parahippocampal hyperactivity [26]. Moreover, THC blocks synaptic plasticity and reduces GABAergic and glutamatergic synapse sensitivity to both cannabinoids and opioids [27]. Both of these responses arise from inhibition of retrograde signalling [28] and disruption of synaptic plasticity in striatal areas [29]. Further explanations could arise from $\mathrm{CB}_{1}$ and $\mathrm{D}_{2}$ receptor heterodimers $[30,31]$ that may fine-tune hedonic reward processing by directly influencing downstream signalling pathways. Activated $\mathrm{CB}_{1} / \mathrm{D}_{2}$ heterodimers signal via Gas [31] to raise intracellular cAMP, balancing inhibition of cAMP from independent $\mathrm{CB}_{1}$ and $\mathrm{D}_{2}$ receptors that signal via Gai. Genetics could also contribute as polymorphisms at the $\mathrm{CNR} 1$ locus (encoding $\mathrm{CB}_{1}$ ) appear to alter the vulnerability to addiction [32].

To delineate the respective roles of cannabinoids and opioids in mediating pleasure, we must breakdown and consider the components of a reward including 'wanting' and 'liking' behaviour [33]. Kent Berridge proposed that dopamine signalling contributes to 'wanting' to seek a reward, but does not contribute to the perceived enjoyment of it [33]. By contrast, as well as the initiating desire, anandamide and drugs that activate $\mu$-opioid receptors appear to enhance the 'liking' component of a reward [20,34]. This provides an elegant explanation to why these three neurotransmitter groups exist in such close proximity at hedonic hotspots. It also allows us to consider how an imbalance of 'wanting' and 'liking' may lead to impulsive behaviour and addiction.

Impulsive behaviour is characterised by a decreased response to threat and an increased urge to seek reward. Genetic variation amongst the population could be valuable to understanding what drives impulsiveness and overstimulates 'wanting' behaviour with a concomitant reduction in 'liking' behaviour. Humans carrying the FAAH C385A missense mutation show increased impulsiveness from increased endocannabinoid signalling in the amygdala and ventral striatum [35]. Dincheva et al. generated knock-in mice with a C385A mutant FAAH allele to reduce FAAH activity and increase anandamide levels in the brain in an attempt to discover the neural pathways responsible for increased impulsiveness [36]. Using anterograde and retrograde tracers in neuroimaging studies, the group uncovered that there was greater connectivity between the basolateral amygdala (BLA) and intralimbic region of the prefrontal cortex (involved in fear extinction) with no change in BLA-prelimbic connectivity (involved in fear acquisition). In this respect, increased endocannabinoid signalling appears to tip the scales from reward seeking behaviour to addictive, compulsive behaviour [37].

\section{Targeting the endocannabinoid system pharmacologi- cally}

Controlled studies evaluating the medical properties of cannabinoids extend back to the 1970s [38]. Unfortunately, promising drug candidates are often stunted by the severity of reported side effects. For example, rimonabant was approved for weight loss before being withdrawn due to reported episodes of depression and suicidal ideation [39]. As such, only a handful of drugs have been licensed for clinical use. Nabilone is a synthetic cannabinoid used as an adjunctive analgesic and antiemetic during chemotherapy [40] and Sativex is a drug preparation containing THC and cannabidiol administered as an oromucosal spray for multiple sclerosis spasticity [41]. Preliminary data suggests that its uses will be extended to treating allodynia and cancer related pain in patients refractory to opioids $[42,43]$.

For decades, opioids have been the drug class of choice in the treatment of severe pain. However, they too carry a burden of undesirable side effects including respiratory depression, tolerance, and dependence [44]. Emerging evidence suggests that cannabinoid and opioid combination therapy could provide the requisite pain relief provided by opioids but with lower doses to eliminate unwanted side effects [45]. One approach progressing through early clinical trials is the co-supression of endogenous endocannabinoid and endogenous opioid (enkephalin mediated) breakdown with FAAH inhibitors and enkephalinase inhibitors [46]. Another promising avenue is co-administration of cannabinoid and opioid agonists. A low dose $\mathrm{CB}_{1}$ agonist co-administered with morphine enhances the antinociceptive property of morphine, crucially, without the discriminative stimulus and positive reinforcement effects of morphine alone [47]. Clinically, this could translate as a reduction of opioid abuse liability. The mechanism behind the findings is unclear but likely to be attributable to the overlapping pharmacodynamic profiles of opioids and cannabinoids. Cannabinoids could mask the effect of opioids or potentiate the release of endogenous opioids [48,49]. There 
could be allosteric modulation at play, off target effects, or opioid and cannabinoid receptor heterodimerisation that is known to exist [50] but the functional consequences of which are yet to be investigated. Moreover, pain does not always manifest as physical suffering; pain can encompass emotional symptoms arising from psychological factors. A specific example is post-traumatic stress disorder (PTSD), which has no established pharmacotherapy. However, synthetic $\mathrm{CB}_{1}$ agonists have been shown to significantly improve the number of sleep hours and reduce the incidence of nightmares in preclinical trials [51]. This provides yet another therapeutic outlet to explore as the role of cannabinoids is more fully understood.

\section{Conclusion}

In $350 \mathrm{BC}$, Aristotle said that emotions are feelings 'attended by pain or pleasure' [20]. 2000 years on, we are beginning to understand how the experiences of pain and pleasure are subject to a conflict between their sensory and affective components.

Pain is notoriously difficult to manage and there is an unmet need for effective drugs that do not result in dependence or tolerance. Similarly, there is scope to improve the manifestations of aberrant reward processing in addiction and impulsiveness for example. Endocannabinoid signalling provides an elegant link between pain and pleasure, and targeting the highly conserved ECS pharmacologically is gaining traction.

Opioids are a mainstay of pain management but co-administration with $\mathrm{CB}_{1}$ agonists facilitates lower dose opioid use with fewer side effects and without compromising the level of pain relief. Endocannabinoids could yet be implicated alongside more basic pain medication including paracetamol that has been shown to blunt the emotional response to both positive and negative stimuli [52]. Furthermore, the contribution of endocannabinoids in the increasingly well-characterised basis of the placebo effect should not be neglected, and could be exploited in managing psychosomatic aspects of pain.

Targeting the endocannabinoid system efficaciously is likely to be difficult until the intricacy and widespread nature of endocannabinoid signalling in the body is better understood. Potential side effects mediated through $\mathrm{CB}_{2}$ cannot be discounted and cannabinoids acting at non- $\mathrm{CB}_{1} / \mathrm{CB}_{2}$ requires greater investigation [53]. Moreover, this review has focused on how the ECS overlaps with opioid and dopaminergic signalling in pain and pleasure processing, but a growing body of evidence implicates cannabinoids interacting with noradrenergic and serotonergic circuits that are central to emotional processing and mood [54,55]. With cannabinoids' extensive role in modulating neurotransmission in the brain and the inextricable link to pain and pleasure pathways, we must now more carefully consider how to reflect the interplay of neural circuits in developing drugs for pain and mood disorders.

\section{Acknowledgements}

I would like to thank Dr. Asif J Iqbal, Dr. Lewis Taylor, and Professor David R Greaves.

\section{References}

1. Leknes S, Tracey I (2008) A common neurobiology for pain and pleasure. Nat Rev Neurosci 9: 314-320. [crossref]

2. Ellingsen DM, Wessberg J, Eikemo M, Liljencrantz J, Endestad T, et al. (2013) Placebo improves pleasure and pain through opposite modulation of sensory processing. Proc Natl Acad Sci U S A 110: 17993-17998. [crossref]

3. Mechoulam R, Hanuš LO, Pertwee R, Howlett AC (2014) Early phytocannabinoid chemistry to endocannabinoids and beyond. Nat Rev Neurosci 15: 757-764. [crossref]

4. Davis MP (2014) Cannabinoids in pain management: CB, CB2 and non-classic receptor ligands. Expert OpinInvestig Drugs 23: 1123-1140.[crossref]

5. Tanasescu R, Constantinescu CS (2010) Cannabinoids and the immune system: an overview. Immunobiology 215: 588-597. [crossref]

6. Cota D (2003) The endogenous cannabinoid system affects energy balance via central orexigenic drive and peripheral lipogenesis. The Journal of Clinical Investigation 11: 423- 431 .

7. Manzanares J (2006) Role of the Cannabinoid System in Pain Control and Therapeutic Implications for the Management of Acute and Chronic Pain Episodes. Current Neuropharmacology 239-257.

8. Devane WA, Dysarz FA, Johnson MR, Melvin LS, Howlett AC, et al. (1988) Determination and characterization of a cannabinoid receptor in rat brain. Mol Pharmacol 34: 605-613.[crossref]

9. Welch SP (2009) Interaction of the cannabinoid and opioid systems in the modulation of nociception. Int Rev Psychiatry 21: 143-151.[crossref]

10. Benedetti F, Thoen W, Blanchard C, Vighetti S, Arduino C, et al. (2013) Pain as a reward: changing the meaning of pain from negative to positive co-activates opioid and cannabinoid systems. Pain 154: 361-367.[crossref]

11. Peciña M, Martínez-Jauand M, Hodgkinson C, Stohler CS, Goldman D, et al. (2014) FAAH selectively influences placebo effects. Mol Psychiatry 19: 385-391.[crossref]

12. Wiech K, Vandekerckhove J, Zaman J, Tuerlinckx F, Vlaeyen JW, et al. (2014) Influence of prior information on pain involves biased perceptual decision-making. Curr Biol 24: 679-681.[crossref]

13. Meagher MW, Arnau RC, Rhudy JL (2001) Pain and emotion: effects of affective picture modulation. Psychosom Med 63: 79-90.[crossref]

14. Peciña S (2008) Opioid reward 'liking' and 'wanting' in the nucleus accumbens. Physiol Behav 94: 675-680.[crossref]

15. Koepp MJ, Hammers A, Lawrence AD, Asselin MC, Grasby PM, et al. (2009) Evidence for endogenous opioid release in the amygdala during positive emotion. Neuroimage 44: 252-256. [crossref]

16. Gardner EL, Vorel SR (1998) Cannabinoid transmission and reward-related events. Neurobiol Dis 5: 502-533.[crossref]

17. Kut E, Candia V, von Overbeck J, Pok J, Fink D, et al. (2011) Pleasure-related analgesia activates opioid-insensitive circuits. $J$ Neurosci 31: 4148-4153.[crossref]

18. Friemel CM (2014)The CB1 Receptor as an Important Mediator of Hedonic Reward Processing. Neuropsychopharmacology 3: 2387-2396.

19. Fattore L, Melis M, Fadda P, Pistis M, Fratta W (2010) The endocannabinoid system and nondrug rewarding behaviours. ExpNeurol 224: 23-36. [crossref]

20. Kringelbach ML, Phillips H (2014)Emotion: pleasure and pain in the brain. Oxford University Press 139-141.

21. Benedetti F (2014) Drugs and placebos: what's the difference?: Understanding the molecular basis of the placebo effect could help clinicians to better use it in clinical practice. EMBO Rep 15: 329-332.[crossref]

22. Volkow ND (2003) Expectation Enhances the Regional Brain Metabolic and the Reinforcing Effects of Stimulants in Cocaine Abusers. Journal of Neuroscience 2: 11461-11468.

23. Gu X (2014) Belief about nicotine selectively modulates value and reward prediction error signals in smokers. PNAS 11: 2539-2540.

24. Castro DC, Berridge KC (2014) Advances in the neurobiological bases for food 'liking' versus 'wanting'. Physiol Behav 136: 22-30.[crossref]

25. Curran HV, Freeman TP, Mokrysz C, Lewis DA, Morgan CJ, et al. (2016) Keep off the grass? Cannabis, cognition and addiction. Nat Rev Neurosci 17: 293-306.[crossref]

26. Roberts GM, Nestor L, Garavan H (2009) Learning and memory deficits in ecstasy users and their neural correlates during a face-learning task. Brain Res 1292: 71-81. [crossref]

27. Hoffman AF (2003) Functional tolerance and blockade of long-term depression at synapses in the nucleus accumbens after chronic cannabinoid exposure. $J$ Neurosci 2: $4815-4820$.

28. Mato S, Chevaleyre V, Robbe D, Pazos A, Castillo PE, et al. (2004) A single in-vivo exposure to delta 9THC blocks endocannabinoid-mediated synaptic plasticity. Nat Neurosci 7: 585-586.[crossref] 
29. Lafourcade M, Elezgarai I, Mato S, Bakiri Y, Grandes P, et al. (2007) Molecular components and functions of the endocannabinoid system in mouse prefrontal cortex. PLoS One 2: e709.[crossref]

30. Kearn CS (2004) Physical interactions of CB1 cannabinoid and D2 receptors. Symposium of the Cannabinoids

31. Kearn CS (2005) Concurrent Stimulation of Cannabinoid CB1 and Dopamine D2 Receptors Enhances Heterodimer Formation: A Mechanism for Receptor Cross-Talk? Molecular Pharmacology 6: 1697-1700.

32. Zhang PW (2014) Human cannabinoid receptor 1: 5' exons, candidate regulatory regions, polymorphisms, haplotypes and association with polysubstance abuse. Mol. Psychiatry 916-931.

33. Berridge KC (1996) Food reward: brain substrates of wanting and liking. Neuroscience and Behavioural Reviews 20: 1-2.

34. Mahler SV (2007) Endocannabinoid Hedonic Hotspot for Sensory Pleasure: Anandamide in Nucleus Accumbens Shell Enhances 'Liking' of a Sweet Reward. Neuropsychopharmacology 3: 2267-2278.

35. Hariri AR (2009) Divergent Effects of Genetic Variation in Endocannabinoid Signaling on Human Threat- and Reward-Related Brain Function. Biol. Psychiatry 6: 9-16.

36. Dincheva I, Drysdale AT, Hartley CA, Johnson DC, Jing D, et al. (2015) FAAH genetic variation enhances fronto-amygdala function in mouse and human. Nat Commun 6 : 6395. [crossref]

37. Melis M, Pistis M (2007) Endocannabinoid signaling in midbrain dopamine neurons: more than physiology? Curr. Neuropharmacology 268-277.

38. Ben Amar M (2006) Cannabinoids in medicine: A review of their therapeutic potential. $J$ Ethnopharmacol 105: 1-25.[crossref]

39. Sam AH, Salem V, Ghatei MA (2011) Rimonabant: From RIO to Ban. J Obes 2011: 432607. [crossref]

40. Dalzell AM, Bartlett H, Lilleyman JS (1986) Nabilone: an alternative antiemetic for cancer chemotherapy. Arch Dis Child 61: 502-505.[crossref]

41. Rog DJ, Nurmikko TJ, Friede T, Young CA (2005) Randomized, controlled trial of cannabis-based medicine in central pain in multiple sclerosis. Neurology 65: 812-819. [crossref]

42. Nurmikko TJ (2007) Sativex successfully treats neuropathic pain characterised by allodynia: a randomised, double-blind, placebo-controlled clinical trial. Pain 13: 210-220.

43. Johnson JR (2013) An Open-Label Extension Study to Investigate An Open-Label Extension Study to Investigate The Long-Term Safety and Tolerability of THC/CBD Oromucosal Spray and Oromucosal THC Spray in Patients With Terminal Cancer-
Related Pain Refractory to Strong Opioid Analgesics. Journal of pain and symptom management 4: 207-218.

44. Scavone JL, Sterling RC, Van Bockstaele EJ (2013) Cannabinoid and opioid interactions: implications for opiate dependence and withdrawal. Neuroscience 248 : 637-654. [crossref]

45. Nadal X, La Porta C, Andreea Bura S, Maldonado R (2013) Involvement of the opioid and cannabinoid systems in pain control: new insights from knockout studies. Eur J Pharmacol 716: 142-157.[crossref]

46. Roques BP, Fournié-Zaluski MC, Wurm M (2012) Inhibiting the breakdown of endogenous opioids and cannabinoids to alleviate pain. Nat Rev Drug Discov 11: 292310. [crossref]

47. Maguire DR (2013) Interactions between $\mu$-Opioid Receptor Agonists and Cannabinoid Receptor Agonists in Rhesus Monkeys: Antinociception, Drug Discrimination, and Drug Self-Administration. JPET 34: 3354-3600.

48. Pacheco Dda F, Klein A, Perez AC, Pacheco CM, de Francischi JN, et al. (2009) Central antinociception induced by mu-opioid receptor agonist morphine, but not delta- or kappa-, is mediated by cannabinoid CB1 receptor. Br J Pharmacol 158: 225-231. [crossref]

49. Valverde $O$ (2001) $\Delta$-9-tetrahydrocannabinol releases and facilitates the effects of endogenous enkephalins: reduction in morphine withdrawal syndrome without change in rewarding effect. Eur. J Neurosci 1:1816-1820.

50. Bushlin I, Gupta A, Stockton SD Jr, Miller LK, Devi LA, et al. (2012) Dimerization with cannabinoid receptors allosterically modulates delta opioid receptor activity during neuropathic pain. PLoS One 7: e49789.[crossref]

51. Ragen BJ, Seidel J, Chollak C, Pietrzak RH, Neumeister A, et al. (2015) Investigationa drugs under development for the treatment of PTSD. Expert OpinInvestig Drugs 24: 659-672.[crossref]

52. Durso GRO, Luttrell A, Way BM (2015) Over-the-Counter Relief From Pains and Pleasures Alike: Acetaminophen Blunts Evaluation Sensitivity to Both Negative and Positive Stimuli. Psychological Science 26: 750-758.

53. Taylor L, Christou I, Kapellos TS, Buchan A, Brodermann MH, et al. (2015) Primary Macrophage Chemotaxis Induced by Cannabinoid Receptor 2 Agonists Occurs Independently of the CB2 Receptor. Sci Rep 5: 10682.[crossref]

54. Burokas A, Martín-García E, Gutiérrez-Cuesta J, Rojas S, Herance JR, et al. (2014) Relationships between serotonergic and cannabinoid system in depressive-like behavior: a PET study with [11C]-DASB. J Neurochem 130: 126-135.[crossref]

55. Carvalho AF, Van Bockstaele EJ (2012) Cannabinoid modulation of noradrenergic circuits: implications for psychiatric disorders. Progress in Neuropsychopharmacology and Biological Psychiatry 3: 59-67.

Copyright: (C2016 Brodermann MH. This is an open-access article distributed under the terms of the Creative Commons Attribution License, which permits unrestricted use, distribution, and reproduction in any medium, provided the original author and source are credited. 